\title{
On developing an automatic control system for the dump conveyor of a wheel excavator
}

\author{
Yakov Liberman and Olga Lukashuk* \\ Ural Federal University, 19 Mira street, 620002, Ekaterinburg, Russia
}

\begin{abstract}
One of the main ways to increase the rotary excavator productivity is to automate excavator operation. The article presents an automatic speed adjustment system for the stacking conveyor belt of a trench rotary excavator. It is shown that the automated control system will allow taking into account the rotor actual rotation speed and the bucket fill factor, processing data and solving tasks of the excavator operation automatic control in excavation mode. The represented automated control system will ensure the conveyor operation with the necessary, but not maximum speed. Therefore, the conveyor will not work "in vain", that, in its turn, will reduce operating costs including energy.
\end{abstract}

\section{Introduction}

Due to intensive building activity development in recent years the zero cycle earthworks volume on buildings and constructions production has significantly increased in our country [1-3]. Such works are often performed using trench rotary excavators (TRE), which are a set of multi-bucket earth-moving machines with a rotating wheel equipped with elements for cutting and lifting soil from the trench with buckets, a tractor, and a stacking conveyor that moves the soil extracted from the trench to a certain area remote from the excavator [4, 5]. The rotary trench excavator is much-in-demand for constructing gas pipelines, sewer and collector utilities, laying underground communication systems for kilometer distances, and digging shallow ditches. It is convenient for excavating earth during excavations or large-scale repairing and rescue operations. Moreover, TRE are used in mining industry, in the field of defense, and land reclamation $[6,7]$.

Increased TRE use causes their operation cost growth, in particular, of the part that is featured by the high energy consumption machines the excavator consists of. Due to this the actual task of modern TRE designing is to reduce their energy consumption [8].

One of the possible ways to solve the task specified may be the stacking conveyor, which is a part of TRE, control automation. Let's consider the theoretical prerequisites for building the automation system that allows to implement this way.

\footnotetext{
* Corresponding author: oldim96@mail.ru
} 


\section{The main part}

It is known [9] the conveyor consumption power $N$ is mainly used on bringing speed to the soil $\left(N_{1}\right)$, rising the soil to the loading height $\left(N_{2}\right)$, the soil friction against the conveyor side flaps, the soil friction against the soil in the flaps areas $\left(N_{3}\right)$, and friction against the conveyor apron $\left(N_{4}\right)$.

The first of these components can be defined as,

$$
N_{1}=\frac{Q \cdot \psi \cdot V_{S}^{2}}{2 \cdot 3600 \cdot 1000}
$$

where $Q$ is the capacity of the stacking conveyor, $\mathrm{m}^{3} / \mathrm{h} ; \psi$ is the volume weight of the soil, $\mathrm{kg} / \mathrm{m}^{3} ; V_{s}$ is the speed of the soil movement acquired by the latter under the friction forces action between it and the conveyor belt, $\mathrm{m} / \mathrm{s}$.

The second component is calculated as follows

$$
N_{2}=\frac{Q \cdot \psi \cdot g \cdot H}{3600 \cdot 1000},
$$

where $g$ is the acceleration of gravity, $\mathrm{m} / \mathrm{s}^{2}, H$ is the height of the soil lifting with the conveyor in the vertical direction, $\mathrm{m}$.

The third component is calculated as follows

$$
N_{3}=\frac{f \cdot Q^{2} \cdot V_{b}}{3600 \cdot 1000},
$$

where $f$ is the soil friction coefficient against the conveyor side flaps steel; $V_{b}$ is the speed of the conveyor belt, $\mathrm{m} / \mathrm{s}$, and the fourth component is calculated as follows

$$
N_{4}=P \cdot V_{b},
$$

where $P$ is the turning effort losses of the conveyor drive on the belt friction against the aprons, $\mathrm{kN}$.

It has been experimentally proved that the value can be defined as

$$
P=1,5 \cdot \frac{Q}{360 \cdot V_{s} \cdot b_{a}},
$$

assuming that is $b_{a}$ a part of the conveyor belt width, not covered with the aprons, equal to $(0.75 \ldots 0.80) b_{b}$, where $b_{b}$ is the belt width, $\mathrm{m}$.

Experiments have also revealed that

$$
V_{b}=\lambda \cdot V_{s}^{\mu}
$$

where $\lambda$ and $\mu$ are the values, the first of which is within 1.0..1.2 and the second one is close to $1[9,10]$. follows

With accuracy sufficient for engineering calculations it is acceptable to express $V_{s}$ as

$$
V_{s}=\frac{V_{b}}{\lambda}
$$

that allows to present $N_{4}$ as follows

$$
N_{4}=1,5 \cdot \frac{Q \cdot \lambda}{360 \cdot b_{a}} .
$$

Replace in (1) with the value from (3) and specify as

$$
\frac{\psi}{2 \cdot 3600 \cdot 1000 \cdot \lambda^{2}}=A
$$

Then $N_{1}=A \cdot Q \cdot V_{b}^{2}$. 
Specify $\frac{\psi \cdot g \cdot H}{3600 \cdot 1000}=B$, and obtain $N_{2}=B \cdot Q$.

Put

$$
\frac{f}{3600 \cdot 1000}=C
$$

and obtain $N_{3}=C \cdot Q^{2} \cdot V_{b}$.

If further we enter the designation $1,5 \cdot \frac{\lambda}{360 \cdot b_{a}}=D$, then we will obtain

$$
N=\eta\left(A \cdot Q \cdot V_{b}^{2}+B \cdot Q+C \cdot Q^{2} \cdot V_{b}+D \cdot Q\right)
$$

where $\eta$ is the efficiency factor that takes into account the power losses in transmission, intermediate supports bearings, and the conveyor tension drum.

It is clear that $N$ is minimal with the minimum of expression $N^{\prime}$ in (6) in parentheses. Find the extremum of this expression from the condition

$$
\frac{d N^{\prime}}{d V_{b}}=2 \cdot A \cdot Q \cdot V_{b}+C \cdot Q^{2}=0
$$

The solution (7) with respect to $V_{b}$ gives

$$
V_{b}=-\frac{C \cdot Q}{2 \cdot A}
$$

The fact that (8) corresponds to the minimum (6) follows from

$$
\frac{d^{2} N^{\prime}}{d V_{b}^{2}}=2 \cdot A \cdot Q>0
$$

If we put the values $C$ from (5) and $A$ from (4) in (8), we will find

$$
V_{b}=-\frac{f \cdot \lambda^{2}}{\psi} \cdot Q
$$

The " - " sign in (9) has a very specific physical meaning that the actions $Q$ and $V_{b}$ are opposite: $Q$ corresponds to the conveyor loading with the soil, and $V_{b}$ - to the unloading.

The solution obtained is found under condition (7), which does not take into account the fact that when transporting the soil by the conveyor, $h_{s}$ - the height of the soil flow on the conveyor may change, $\mathrm{m}$. That means it is true for the cases when TRE is operated in modes providing low productivity, when fluctuations of $h_{s}$ do not result in spilling the soil from the conveyor belt and do not matter in principle. But if it matters, $h_{s}$ should be permanent. In such a case the expression (2) should not use (3), but an empirical formula

$$
V_{s}=\frac{Q}{3600 \cdot b_{a} \cdot h_{s}} \text {. }
$$

If we put $V_{s}$ from it into (2), we will obtain $P=1,5 \cdot 10 \cdot h_{s}$. Then specify $1,5 \cdot 10=$ $E, N_{4}=E \cdot h_{s} \cdot V_{b}$ and

$$
\left.N^{\prime}=A \cdot Q \cdot V_{b}^{2}+B \cdot Q+C \cdot Q^{2} \cdot V_{b}+E \cdot h_{s} \cdot V_{b}\right)
$$

Whereas, the minimum $N^{\prime}$ will be provided

$$
\frac{d N^{\prime}}{d V_{b}}=2 \cdot A \cdot Q \cdot V_{b}+C \cdot Q^{2}+E \cdot h_{s}=0,
$$

from which

$$
V_{b}=-\frac{C \cdot Q^{2}+E \cdot h_{s}}{2 \cdot A \cdot Q}
$$


This expression is valid for the specified $h_{s}$. If we put $C, A$, and $E$ here, we will finally obtain

$$
V_{b}=-\frac{\left(\frac{f \cdot Q^{2}}{3600 * 1000}+1,5 \cdot 10 \cdot h_{S}\right) \cdot 3600 \cdot 1000 \cdot \lambda^{2}}{\psi \cdot Q}=-\frac{f \cdot Q^{2} \cdot \lambda^{2}+3600 \cdot 15000 \cdot h_{S} \cdot \lambda^{2}}{\psi \cdot Q} .
$$

When designing a TRE, its rotor, and a stacking conveyor, today, as a rule, they take $Q=Q_{\max }=F \cdot V_{\max }$, where $F$ is the trench section area, $V_{\max }$ is the maximum speed of the excavator linear movement (feed).

But under real conditions $Q \neq Q_{\max }$, but depends on the actual speed of the rotor rotation $n$, and the rotor bucket fill factor

$$
K_{f}=\frac{C_{0} \cdot z}{2 \cdot \pi \cdot R}
$$

where $z$ is the rotor buckets number, $R$ is the rotor radius at the soil gravity center in the bucket, $C_{0}$ is the excavator feed to the bucket $\left(C_{0} \cdot z\right.$ is feed to the rotor revolution).

Actual

$$
Q=60 \cdot q \cdot z \cdot n \cdot \frac{K_{f}}{K_{p}}
$$

where $q$ is the bucket capacity, $n$ is the rotor rotation frequency, $K_{p}$ is the coefficient of the soil loosening $[11,12]$.

Now put (11) into (12), and (12) into (9) or into (10), and depending on $n$ and $C_{0}$ we can find $V_{b}$. Based on this it is possible to build a conveyor control automation system.

This system is a speed adjustment system of the TPE stacking conveyor belt, and is shown in Fig. 1. It includes device 1 for specifying the TRE rotor rotation speed $n$ via speed controller 2 connected with the rotor drive 3, the device 4 for the excavator feed specifying $C_{0}$ to the bucket via speed controller 5 connected with the tractor drive 6 , the input unit 7 of the design parameters $R, z$, and $q$ the rotor, and the unit 8 for calculating the excavator actual productivity $Q$, block 9 of the belt speed $V_{s}$ calculation via speed controller 10 connected to the conveyor drive 11 , and the system adjustment unit 12 , which is used to enter the values $K_{p}, \lambda, \psi, f_{p}$ and, if necessary, $h_{s}$, into the system.

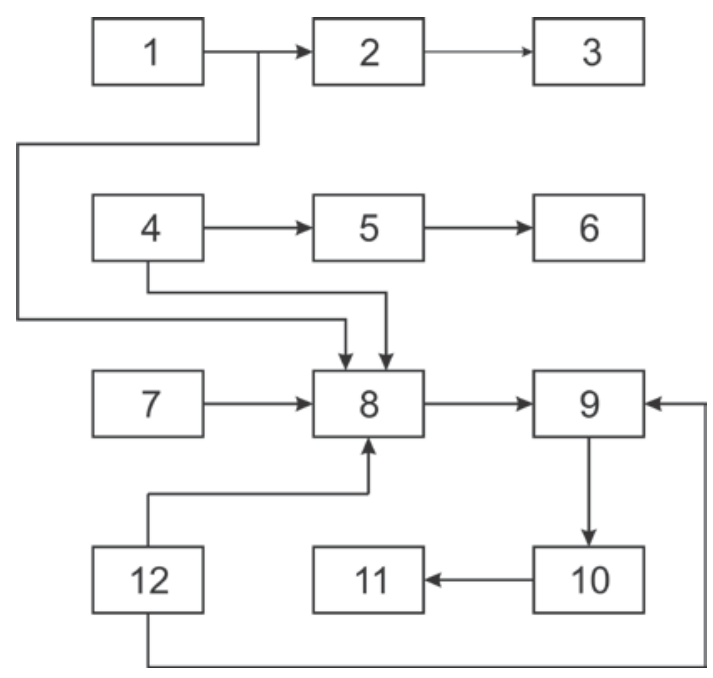

Fig. 1. The flow chart of the TRE stacking conveyor belt speed automatic adjustment. 


\section{Conclusion}

When being controlled by this system, every time the conveyor will run with the required speed, but not the maximum speed, therefore, it will not work "in vain". Its wear and tear will decrease, its durability will increase, and its operating costs, including energy costs, will decrease.

\section{References}

1. E.M. Kudryavtsev, Complex mechanization of construction (Moscow: ASV Publishing house, 2005)

2. A.S. Shackij, To a question of rotor trench excavators in domestic pipeline construction using, J. Pipeline transport:theory and practice, v. 2(36), pp. 28-31 (2013)

3. A.I. Docenko, G.I. Karasev, I.K. Shestopalov, Earthmoving machines (Moscow: Bastet, 2012)

4. D.I. Fedorov Working bodies of earth-moving machinery (Moscow: Engineering, 1977)

5. S. S. Dobronravov, Construction machines and equipment: Handbook (Moscow: Higher school, 2006)

6. V. M. Vladimirov, V. K. Trofimov, Improving the productivity of multi-bucket excavators (Moscow: Nedra, 1980)

7. R.Yu. Poderni Mechanical equipment of quarries: Textbook for higher education institutions, (Moscow: MGSU publishing House, 2007)

8. V.B. Permyakov, S.M. Kuznetsov, Assessment of organizational and technological reliability of construction machines, J. Mechanization of construction, 11, pp. 24-29 (2008)

9. B.N. Abramov, O.A. Lukashuk, Multi-Bucket excavators: design and calculation, (Yekaterinburg: URFU, 2012)

10. M. I. Shchadov, V. M. Vladimirov, V. V. Guzhovsky, Handbook of mechanics of open works. Excavation-transport machines of continuous action, (Moscow: Nedra, 1989)

11. M.N. Goldstein, Mechanical properties of soils: Stress-strain and strength characteristics (Moscow: Stroyizdat, 1979)

12. Yu.A. Vetrov, Soil cutting by earth-moving machinery (Moscow: Engineering, 1971) 